\title{
SOME SOBER CONCEPTIONS OF MATHEMATICAL TRUTH
}

\author{
Marco Panza \\ REHSEIS, CNRS and University of Paris 7.
}

\begin{abstract}
It is not sufficient to supply an instance of Tarski's schema, " $p$ " is true if and only if $p^{1}$ for a certain statement in order to get a definition of truth for this statement and thus fix a truth-condition for it. A definition of the truth of a statement $x$ of a language $L$ is a bi-conditional whose two members are two statements of a meta-language $L^{\prime}$. Tarski's schema simply suggests that a definition of truth for a certain segment $x$ of a language $L$ consists in a statement of the form: ${ }^{\lceil} \boldsymbol{v}(x)$ is true if and only if $\tau(x)^{\rceil}$, where ${ }^{\lceil} v(x)^{\rceil}$is the name of $x$ in $L^{\prime}$ and $\tau(x)$ is a function $\tau: S \rightarrow S^{\prime}$ ' $\left(S\right.$ and $S^{\prime}$ 'being the sets of the statements respectively of $L$ end $L^{\prime}$ ) which associates to $x$ the statement of $L^{\prime}$ expressed by the same sentence as that which expresses $x$ in $L$. In order to get a definition of truth for $x$ and thus fix a truth-condition for it, one has thus to specify the function $\tau$. A conception of truth for a certain class $X$ of mathematical statements is a general condition imposed on the truth-conditions for the statements of this class. It is advanced when the nature of the function $\tau$ is specified for the statements belonging to $X$. It is sober when there is no need to appeal to a controversial ontology in order to describe the conditions under which the statement $\tau(x)$ is assertible. Four sober conceptions of truth are presented and discussed.
\end{abstract}

Key words: mathematical truth, conception of truth, truth-condition, statements vs. sentences, Tarski's condition.

Truth is generally considered to be a crucial matter in the philosophy of mathematics. It is quite common to define realism in mathematics as the thesis that mathematical statements can be true, but their (eventual) truth does not depend on the fact that they are proved, or even that they could be proved in our mathematical theories, being rather somehow independent of us. In other words, it is generally accepted that to be realist in mathematics means to consent to the thesis that truth or falsehood are intrinsic properties of mathematical statements.

I do not intend to discuss this thesis here. My aim is much more modest. I simply observe that, commonly, this definition assumes implicitly not only that there are mathematical statements, but also that they are homogeneous with respect to the property of them that makes them eventually true or false. This means that mathematical statements, whether true or false, are all so in the same sense or for the same sort of reasons. If one does not take this for granted but nevertheless accepts the previous definition, one can hardly admit that realism in mathematics is a welldefined and even a consistent thesis, and thus argue in favour of or against it. One evidence for this is that people who consider themselves to be realist in mathematics in the previous sense mainly look for a suitable conception of mathematical truth, and only for one.

When mathematics is considered abstractly as a (more or less well-defined) domain of justified beliefs, or even as a system of statements of a certain sort that belong to such a domain because of their form, their content, or the modality of their

1

M. H. G. Hoffmann, J. Lenhard, F. Seeger (ed.), Activity and Sign-Grounding Mathematics Education. Festschrift for Michael Otte, 1 - ?.

(C) 2004 Kluwer Academic Publishers. Printed in the Netherlands. 
justification, such an assumption is quite tenable. Explicitly or not, it can even enter the definition of mathematics itself and, when the realist thesis (as previously defined) is accepted, also contribute to the definition of mathematical knowledge. In contrast, it seems to me that when mathematics is considered as an actual practice or activity accompanying the history of humanity, or even as the system of issues resulting from such a practice or activity, this same assumption is quite doubtful. There is no doubt, I think, that from such a point of view, one may rightly speak about mathematical statements, that is, admit that the term "mathematical statement" refers to genuine objects. And I also hold that one can sensibly assign to these latter objects the property of being true. But, when mathematics is conceived in such a way, there is no guarantee that these objects are homogeneous with respect to what makes they are true or false; there is no guarantee that, if true, they are all so in the same sense or for the same sort of reasons.

There are at least two ways to argue that this is not really the case. The first one consists in seeking out various occurrences of the term "true" and their cognates in texts unanimously considered as mathematical ones, and to show, by means of textual analysis, that this term does not have the same sense across these occurrences. The problem with this strategy is that the argument it provides could be countered by arguing that, although they occur in mathematical texts, some of these occurrences are not specifically mathematical. Thus, I prefer to look beyond this objection and to proceed in the second way. I shall present different senses in which it seems to me that one should admit that a mathematical statement could be true, and argue that when a statement is true according to one of these senses it is not true for the same sort of reason as when it — or any other statement—is true according to any other one of them.

In doing this, my intention is not to argue against realism in mathematics. Far from it, I argue that if the opposition between realism and anti-realism in mathematics is genuine and crucial, and if one speaks of mathematical truth in the senses I shall consider here, then this opposition is not concerned with truth. This is the reason why I take these senses to be sober ones. My aim is simply to show that it is possible to speak of truth in mathematics without being engaged in doubtful and controversial ontology. Of course, I admit that one could also speak of truth in mathematics in other and not sober senses. It is even a matter of fact that a lot of philosophers and mathematicians have done and do this. I do not intend to make a stand against their attitude here. I simply advance the hypothesis that sober senses (the ones I shall consider or some other ones that could be added to my list) would suffice to give a satisfactory account of mathematical practice or activity (though I concede that this account might not meet the intentions and metaphysical convictions of some mathematicians).

Up to now, I have generically used the term "sense" in order to refer to a certain way to conceive truth in mathematics. To be more precise, I shall from now on speak of truth-conditions and of definitions and conceptions of truth. I shall speak of truth- 
condition and definition of truth for a certain statement in order to refer respectively to the condition that such a statement has to satisfy in order to be true, and to the statement that specifies such a condition. I shall speak instead of conception of truth for a certain class of statements in order to refer to a general condition imposed on the truth-conditions for the statements of this class. Thus, in order to present different senses in which one should admit that mathematical statements could be true, I shall advance different conceptions of truth for different classes of mathematical statements.

Before doing this, let me present a general condition that, in my opinion, any definition of truth for a mathematical statement, should satisfy.

This is Tarski's condition. I require that any definition of the truth of a mathematical statement should be expressed by an instance of Tarski's schema: [" $p$ " is true if and only if $p$. I shall not argue in favour of such a condition. I simply argue that, taken as such, an instance of Tarski's schema is nothing but a sentence of a certain language, i.e. a well formed combination of terms (or formula) of this language. It is thus not sufficient to supply an instance of Tarski's schema for a certain statement in order to get a definition of truth for this statement and thus fix a truth-condition for it. In order to do that, it is also necessary to interpret such a sentence, that is, to take it as being the expression of a certain statement.

Suppose that a particular instance of Tarski's schema is uttered in a certain language $L^{\prime}$ in order to provide a truth-condition for a certain statement $x$ of another language $L$. $L^{\prime}$ 'should then serve as a meta-language with respect to $L$. Hence $L$ ' would contain a name for $x$, to be used to form the first member of the bi-conditional constituting such an instance of Tarski's schema. This name should not only denote $x$ in $L$ ', but also be, as such, functionally related to the second member of this biconditional. And this second member should, in turn, be a sentence of $L$ ' expressing a certain statement. It is just this latter statement that fixes a truth-condition of $x$.

A definition of the truth of a statement $x$ of a language $L$ is thus a bi-conditional whose two members are two statements of a meta-language $L^{\prime}$, the first saying that $x$ has the property to be true, and the second being the value taken by a function $\psi: N^{\prime} \rightarrow S^{\prime}$ (in which $N^{\prime}$ is the set of the names of $L^{\prime}$ and $S^{\prime}$ the set of the statements of $L^{\prime}$ ) when its argument is the name of $x$ in $L^{\prime}$. Of course, not every name of $L^{\prime}$ could be an argument for such a function. In order to be so, a name of $L$ ' should be the name of a statement of $L$ and result from a quotation. Tarski's schema would then suggest the following: Take the statement $x$ as it is uttered in $L$; transform it into its same name in $L$ ' by adding quotation marks to the sentence of $L$ which expresses it, and use this name to form a statement of $L^{\prime}$ 'saying that $x$ has the property of being true; disquote such a sentence in order to obtain another statement of $L^{\prime}$; and form a bi-conditional whose two members are given by these two statements of $L^{\prime}$. This means that Tarski's schema would suggest to define a function $\tau: S \rightarrow S^{\prime}$ ' $(S$ being the set of the statements of $L$ ) by composing a function $v: S \rightarrow N^{\prime}$, giving the name of $x$ in $L^{\prime}$ when applied to $x$, with another function $\psi: N^{\prime} \rightarrow S^{\prime}$. It is just the value of this function $\tau$ that ultimately would provide a truth-condition for $x$. 
Suppose now that the function $v$ is simply the quotation function: applied to a statement of $L$, it gives a name for this statement in $L$ ' by simply adding quotation marks to the sentence which expresses this statement. Then the function $\psi$ can not simply be the disquotation function, since when this function is composed with $v$, it can only produce the identity function and not a function $\tau$ from $S$ to $S$ '. Thus, either $v$ is not simply the quotation function, or $\psi$ is not simply the disquotation function. It follows that either there is more in Tarski's schema that the simple allegation of the quotation and the disquotation functions (jointly with the instruction to form a suitable bi-conditional by using their values), or it is not sufficient to supply an instance of Tarski's schema for a certain statement in order to get a definition of truth for this statement and thus fix a truth-condition for it. But, in Tarski's schema, there is nothing more than that; hence, it is not sufficient to apply this schema to a certain statement in order to get a definition of truth for this statement and thus fix a truth-condition for it.

A definition of truth of a certain statement $x$ of a language $L$ is rather a biconditional statement of a meta-language $L$ ', whose first member says of $x$ that it has the property of being true, and the second one is the value taken by a function $\tau$ from $S$ to $S^{\prime}$ when it is applied to $x$, under the condition that this function results from the composition of the quotation function $v$ from $S$ to $N^{\prime}$ and a function $\psi$ from $N^{\prime}$ to $S^{\prime}$ such that $\psi(v(x))$ is the statement of $L^{\prime}$ expressed by the same sentence that expresses $x$ in $L$. This bi-conditional statement has thus the form: ${ }^{\lceil} v(x)$ is true if and only if $\tau(x)^{\rceil}$, where ${ }^{\lceil} v(x)^{\rceil}$is the name of $x$ in $L^{\prime}$ and $\tau(x)=\psi(v(x))$.

Of course, a definition of this sort can only be uttered in $L^{\prime}$ if this language contains all the terms of $L$ entering in $x$. This is an obvious necessary condition. But it is not sufficient, since to say that a statement of $L^{\prime}$ is expressed by the same sentence that expresses it in $L$ is not sufficient in order to identify this statement. The work of the function $\psi$ is just to identify this statement.

Thus, either it is admitted that at least some ones of the sentences of $L$ ' are such that one of them may express different statements, or it is admitted that the statements of $L$ ' are not given independently of the specification of the functions $\psi$ and $\tau$. I favour the second possibility. After all, it seems to me very natural to conceive the language $L$ ' as being constructed on the basis of $L$ just in order to utter truth-conditions for the statements of this latter language, rather than as an already given language fortuitously satisfying all the conditions that should be satisfied by a meta-language with respect to $L$ where a definition of truth for a statement of $L$ could be uttered.

It follows that, in my opinion, in order to advance a conception of truth for a certain class $X$ of mathematical statements of a certain language $L$, one should simply specify the nature of the function $\tau$ entering the truth-conditions of the statements belonging to $X$ and allowing the determination of the statements of $L$ '. This is what I shall do.

Before to do that, let me consider some consequences of Tarski's condition. Suppose that one has specified the nature of the function $\tau$ entering the truthconditions of the statements belonging to a certain class $X$ of mathematical 
statements, and has thus advanced a conception of truth for this class of mathematical statements. One can then advance a conception of falsehood in the same way, by assuming that a definition of the falsehood of a certain mathematical statement $x$ belonging to a certain language $L$ and a certain class $X$ of mathematical statements is a bi-conditional statement of a language $L$ ' working as a metalanguage with respect to $L$, namely the statement: $\ulcorner v(x)$ is false if and only if non $\tau(x)^{1}$, where ${ }^{[n o n} \tau(x){ }^{1}$, is the negation of the statement ${ }^{[} \tau(x)^{\top}$ in $L^{\prime}$. It is then only a question of propositional logic to derive in $L$ ' the following bi-conditional: $\lceil v(x)$ is not true if and only if $v(x)$ is falsel. But what about the truth or the falsehood of the negation of $x$ in $L$ ? If such a negation belongs to $X$, the previous definitions give, by substitution: $\left\lceil\nu(\operatorname{non} x)\right.$ is true if and only if $\tau(\text { non } x)^{\rceil}$and $\lceil\boldsymbol{v}($ non $x)$ is false if and

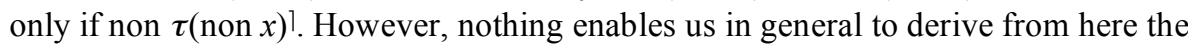
bi-conditionals: ${ }^{\lceil} \boldsymbol{v}($ non $x)$ is true if and only if non $\tau(x)^{\rceil}$, or ${ }^{\lceil} \boldsymbol{v}(x)$ is false if and only

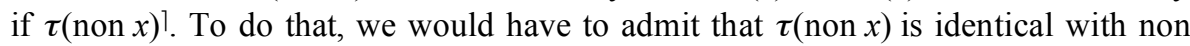
$\tau(x)$, and nothing enables us to admit that in general. Nevertheless, nothing prevents the function $\tau$ from satisfying such a further condition. If this is the case, it is then only a question of propositional logic to derive the bi-conditional ${ }^{\lceil} v($ non $x)$ is true if and only if $v(x)$ is false', and, if either the logic in $L$ or the logic in $L$ ' are classical, also the conditional $\left\lceil v(\right.$ non $x)$ is false if and only if $v(x)$ is true ${ }^{\top}$. It would follow that the truth of the negation of $x$ is equivalent to the falsehood of $x$, and if either the logic in the language which $x$ belongs to or the logic in the language in which this definition is stated are classical, it is also such that the truth of $x$ is equivalent to the falsehood of the negation of $x$. This is a remarkable condition, and it seems to me that we are entitled to qualify a conception of truth that satisfies it as a correspondentist conception of truth.

The first conception of truth I shall advance is just a sober correspondentist conception. Thus it constitutes an example proving that a correspondentist conception of truth for a class of mathematical statements can be sober.

\section{1}

Take a sentence like the following one: "the primitive of $\frac{5 x^{2}+4 a x}{2 \sqrt{a x+a^{2}}}$ is $\frac{x^{2}}{a} \sqrt{a x+a^{2}}+C$." It seems to me that one possible way of understanding the statement expressed by such a sentence, say $s$, is to take it as referring to the symbolic expressions that enter it. If so, the statement $s$ tells us that if one applies the derivative algorithm to the expression $\frac{x^{2}}{a} \sqrt{a x+a^{2}}+C$, one obtains the 
expression $\frac{5 x^{2}+4 a x}{2 \sqrt{a x+a^{2}}}$. It is thus a statement describing a relation between two symbolic expressions, that is, two equivalence-classes of empirical objects such as concrete signs. One is then entitled, I think, to take $s$ as true, just because the application of the derivative algorithm to the expression $\frac{x^{2}}{a} \sqrt{a x+a^{2}}+C$ gives the expression $\frac{5 x^{2}+4 a x}{2 \sqrt{a x+a^{2}}}$. This is simply a way to understand the term "primitive." Let us suppose that $s$ belongs to a language $L$. This means that $L$ is the language in which the sentence "the primitive of $\frac{5 x^{2}+4 a x}{2 \sqrt{a x+a^{2}}}$ is $\frac{x^{2}}{a} \sqrt{a x+a^{2}}+C$ " expresses $s$. The function $\tau$ entering a possible truth-condition of $s$ should thus simply associate $s$ to a statement $\tau(x)$ of a suitable meta-language $L^{\prime}$ expressed in such a language by the same sentence "the primitive of $\frac{5 x^{2}+4 a x}{2 \sqrt{a x+a^{2}}}$ is $\frac{x^{2}}{a} \sqrt{a x+a^{2}}+C "$ and asserting here that the application of the derivative algorithm to the expression $\frac{x^{2}}{a} \sqrt{a x+a^{2}}+C$ gives the expression $\frac{5 x^{2}+4 a x}{2 \sqrt{a x+a^{2}}}$. In other terms, this function should associate $s$ to a statement of $L^{\prime}$ telling us the same as that which the statement $s$ tells us in $L$.

It is easy to generalise this example. Let us consider a class $X$ of mathematical statements of a language $L$ asserting that a certain rule of formal transformation applied to a certain expression produces or does not produce another given expression. A possible conception of truth for such a class of statements simply requires that the function $\tau$ defined over this class associates any statement $x$ of $X$ to a statement of $L$ ' telling us the same as that which the statement $x$ tells us in $L$; that is, that the rule of formal transformation that $x$ refers to, when applied to the first expression that $x$ refers to, produces the second expression that $x$ refers to. I suggest that a lot of mathematical statements can be conceived as asserting that a certain rule of formal transformation applied to a certain expression produces or does not produce another given expression, and that one is thus entitled to apply to them such a correspondentist conception of truth. 


\section{2}

To pass to other conceptions of truth, let us consider the same sentence as before: "the primitive of $\frac{5 x^{2}+4 a x}{2 \sqrt{a x+a^{2}}}$ is $\frac{x^{2}}{a} \sqrt{a x+a^{2}}+C$." One could also understand the statement expressed by such a sentence, say $s^{*}$, by referring the term "primitive" not to the derivative algorithm, but to the general definition of derivative. If so, $s^{*}$

tells to us that the limit of

$$
\frac{\frac{(x+h)^{2}}{a} \sqrt{a(x+h)+a^{2}}+C-\frac{x^{2}}{a} \sqrt{a x+a^{2}}-C}{h}
$$

when $h$ tends toward 0 is equal to $\frac{5 x^{2}+4 a x}{2 \sqrt{a x+a^{2}}}$. If the derivative algorithm is taken as

being justified by having shown that it satisfies the general definition of derivative, the difference between the statement $s^{*}$ and the statement $s$ considered in the previous example is far from being essential from a mathematical point of view. This is not my point, however. What is important for me is that the equivalence of these two statements has to be set up by means of a proof, which is not, as such, part of these two statements. It seems to me that this allows us to consider these two statements as distinct from each other.

$$
\frac{\frac{(x+h)^{2}}{a} \sqrt{a(x+h)+a^{2}}+C-\frac{x^{2}}{a} \sqrt{a x+a^{2}}-C}{h} \text { when } h \text { tends toward } 0 \text { is }
$$

equal to $\frac{5 x^{2}+4 a x}{2 \sqrt{a x+a^{2}}}$ is nothing but asserting that the first expression can be

transformed into the second one by applying certain rules of transformation in a suitable way. If so, the situation does not change with respect to the case considered in Section 2.1: Though the truth of the statement $s$ and the truth of the statement $s^{*}$ do not depend on the same reason, the reasons they depend on are of the same sort; and the truth-conditions of these statements satisfy the same conception of truth, since a certain sequence of rules of transformation is itself a rule of transformation. But one could also argue that this is not so: that when speaking of limit, we are implicitly referring to functions, whereas when operating according to certain rules of transformation, we are working with symbolic expressions, eventually with the symbolic expressions expressing these functions. When we are considering particular functions like the previous ones, the difference between a function and the expression that expresses it could appear to be minor. But it is not certainly so in general, and it is not so in particular, when we are referring to a class of functions respecting certain general conditions, like continuous functions or limited functions. Thus, it should be clear that a statement referring to these functions can not be 
understood as asserting that a certain rule of formal transformation applied to a certain expression produces or does not produce another given expression.

Hence, if one wanted to understand the statement $s^{*}$, or any other statement about functions, as a description of some objects and/or their relations, one should admit that these objects do not simply consist of equivalence-classes of empirical objects. This is also the case for statements about numbers, sets, algebraic structures, and a lot of other mathematical entities that cannot be understood as equivalenceclasses of empirical objects. One could think that there is no way to admit that these statements can be true or false unless their truth-conditions depend on a function $\tau$ that is supposed to associate any one of these statements to a statement of a suitable meta-language telling us that the objects it refers to have the properties or relations it assigns to them. A similar conception of truth is hardly a sober one. Far from arguing here that a similar conception of truth is not tenable, I limit myself to ignoring it and advancing three sober alternative options.

\section{2 .1}

The first two options consist in requiring that the function $\tau$ associates any statement $x$ of the considered class of statements of a language $L$ to a statement of a suitable meta-language $L^{\prime}$, telling us respectively that the statement $x$ has been proved or is provable within the theory to which it belongs.

Of course, to say that $x$ has been proved in a certain mathematical theory is not the same thing as saying that it is provable in such a theory, whatever our notion of proof. I shall not enter into this distinction here. I simply observe that in the first case, $\tau(\operatorname{non} x)$ is certainly not identical with non $\tau(x)$, whereas in the second case, the identity of $\tau(\operatorname{non} x)$ and non $\tau(x)$ depends on the nature of the mathematical theory to which $x$ belongs. Thus, the conception of truth depending on the first option is never correspondentist, whereas the conception of truth depending on the second is not so in general.

However, if the first option were accepted, then it would be very unsatisfactory to define the falsehood of $x$ by the bi-conditional ${ }^{\ulcorner} v(x)$ is false if and only if non $\tau(x)^{\rceil}$. The bi-conditional $\left\lceil\nu(x)\right.$ is false if and only if $\tau(\text { non } x)^{\rceil}$would be preferable. Hence, it would be only a question of propositional logic to derive in $L^{\prime}:{ }^{\lceil} \boldsymbol{v}(\operatorname{non} x)$ is true if and only if $v(x)$ is false ${ }^{\top}$. By substitution, one also would have: ${ }^{\top} v(\operatorname{non} x)$ is false if and only if $\tau$ (non non $x$ ) ${ }^{1}$. Thus, if the logic in $L$ were classical, it would also be only a question of propositional logic to derive in $L$ ': ${ }^{\top} v($ non $x)$ is false if and only if $\tau(x)^{\rceil}$and thus ${ }^{\lceil} v(x)$ is true if and only if $v(\operatorname{non} x)$ is false ${ }^{\top}$. But it would not be possible to derive: $\lceil\boldsymbol{v}(x)$ is not true if and only if $v(x)$ is false $\rceil$. Thus a statement $x$ could be not true, without being false.

One could object to these conceptions of truth by drawing on a classic argument first presented by Tarski. Using godelisation, one can associate injectively each sentence of a formal theory, that is, a mathematical one, to a natural number, and thus each set of sentences of a formal theory to a set of natural numbers. Supposing that any sentence of a formal theory is associated bijectively which a statement that is expresses, and that to prove a statement in such a theory is nothing but to derive in 
it the sentence that expresses it, one could then wonder whether the set of numbers associated to provable statements and the set of numbers associated to true statements are identical. Now, because of the nature of a formal theory, the first of these sets can be characterized in terms of simple arithmetical operations and relations. One could then translate the definition of provability into the language of the theory, that is the object-language. If a similar translation were also possible for the definition of truth, this language would be semantically universal and then it would be possible to use it to formulate the antinomy of the liar. Thus, either the antinomy of the liar can be formulated in the language of a formal theory, or provability within this theory and truth of its statements are not extensionally equivalent properties.

Though admitting that a mathematical theory could take the form of a formal theory and that a proof of a statement in such a theory reduces to a formal deduction of a sentence, this argument does not seem to me to be a conclusive reason to reject the previous conceptions of truth for the statements of such a theory. After all, these are sober conceptions, and they are simply supposed to correspond to two different senses in which mathematicians speak or have spoken of truth. Thus, if Tarski's argument is correct, it simply discloses that these senses make it possible to formulate the antinomy of the liar within a mathematical formal theory. Notice, moreover, that this is not the same as asserting that this theory is not consistent, since the theory itself is completely independent of the nature of the meta-language used to assign to its statements the property of being true.

\section{2 .2}

The comparison between the statements $s$ and $s^{*}$ expressed by the same sentence in a language $L$ suggests the possibility of defining the truth of one of these statements, say $s$, by means of a statement of a meta-language $L^{\prime}$, telling us in this latter language the same as that which the other statement, say $s$, tells us in $L$. In such a way, one could also assign a sober sense to the distinction between truth and proof or provability. One could admit that in order to prove that the primitive of $\frac{5 x^{2}+4 a x}{2 \sqrt{a x+a^{2}}}$ is $\frac{x^{2}}{a} \sqrt{a x+a^{2}}+C$, one should show that the limit of $\frac{\frac{(x+h)^{2}}{a} \sqrt{a(x+h)+a^{2}}+C-\frac{x^{2}}{a} \sqrt{a x+a^{2}}-C}{h}$ when $h$ tends toward 0 is equal to $\frac{5 x^{2}+4 a x}{2 \sqrt{a x+a^{2}}}$, though maintaining that the statement $s^{*}$ is true because the application of the derivative algorithm to the second of these expressions simply produces the first. When understood in this way, the distinction between truth and proof or provability reduces to a methodological one and just concerns the internal organisation of a certain mathematical theory.

A similar view can be applied to universal statements. 
Let us take the example of the last theorem of Fermat. This tells us that if $n$ is a natural number greater than 2 , then there is no trio of strictly positive natural numbers $x, y$, and $z$ such that $x^{n}+y^{n}=z^{n}$. The simpler way to understand this theorem is to take it as assuring us that no substitution of the letters " $x$," " $y$," " $z$ " and " $n$ " in the sentence " $x^{n}+y^{n}=z^{n}$ " with symbols of natural numbers satisfying the previous conditions transforms this sentence into numerical identity. However, there is no way to prove it by considering such substitutions. To do that, it should be rather showed that the fact that $x, y, z$, and $n$ are four natural numbers satisfying these conditions is a sufficient condition for the sum $x^{n}+y^{n}$ to be different from $z^{n}$.

The same point can be made in general. Any universal theorem can be understood in at least two ways: a distributive or extensional way and a compact or intensional way. Extensionally, it asserts that every single element of a certain domain has a certain property; intensionally, it asserts that it is sufficient to belong to such a domain to have this property. If the domain is an infinite one, there is no other way to prove this theorem than by proving the latter. However, one should maintain that this theorem is true because of the former.

In general, one could identify a theorem (of a certain sort) with an equivalenceclass of statements composed of two statements, and assume that the proof of this theorem is concerned with one of these statements, while its truth is concerned with the other.

It would then be sufficient to treat an equivalence-class of statements as a statement whose utterance is nothing but the utterance of one of its members in order to be able to present a conception of truth that fits with such a view. Let us consider a class $X$ of mathematical statements of a language $L$ and two relations of equivalence defined over $X$, say $R^{\prime}$ and $R$ ', such that $R$ ' divides $X$ into several equivalence-classes, each of which is composed of two distinct statements, and $R$," divides $X$ in two equivalence-classes respectively composed of one and only one of the two statements making up each one of the classes of equivalence in which $\mathrm{X}$ is shared by $R$ '. Let $X_{p}$ and $X_{t}$ be the two classes in which $\mathrm{X}$ is divided by $R$ '. A conception of truth for $X$ could require that the function $\tau$ defined over this class associates any statement $x$ of $X$ to a statement of a suitable meta-language $L$ ' telling us the same as the statement of $L$ equivalent to $x$ according to $R$ ' and belonging to the class $X_{t}$. At the same time, one could suppose that in order to prove any statement $x$ of $X$, one should produce an argument showing that the things are as it is said by the statement of $L$ being equivalent to $x$ according to $R$ ' and belonging to the class $X_{t}$.

In the case of the last theorem of Fermat, the role of the statement $x$ is taken by any one of the two statements telling us respectively that: no substitution of the letters " $x$," " $y$," " $z$," and " $n$ " in the sentence " $x^{n}+y^{n}=z^{n}$ " with symbols of naturals numbers satisfying the given conditions transforms this sentence into a numerical identity; and that the fact that $x, y, z$, and $n$ are four natural numbers satisfying these condition is a sufficient condition for the sum $x^{n}+y^{n}$ to be different from $z^{n}$. The role of the statement of $L$ equivalent to $x$ according to $R^{\prime}$ and belonging to the class $X_{t}$ is taken by the first of these two statements, and the role of the statement of $L$ 
equivalent to $x$ according to $R^{\prime}$ and belonging to the class $X_{p}$ is taken by the second one.

If $X$ is a class of universal statements, the relations $R^{\prime}$ and $R$ ' 'can be defined on this class in such a way that $R$ ' associates one to each other two statements telling us respectively that every single element of a certain domain has a certain property, and that it is sufficient to belong to such a domain to have this property, while $X_{t}$ and $X_{p}$ coincide respectively with the class of the elements of $X$ having an extensional form and the class of the elements of $X$ having an intensional form. It is then possible to generalise to any statement of $X$ the previous way to fix the truth-condition of the last theorem of Fermat. However, this is nothing but a particular case of the conception of truth I am presenting here, since nothing prevents us from defining the relations $R$ ' and $R$ ', on a certain class of mathematical statements, either universal or not, in a different way. The example of the statements $s$ et $s^{*}$ suggests a way to do it in a certain case. Other strategies could be followed in other cases.

I shall not enter into a discussion of these strategies. I limit myself to observing that such a conception is a correspondentist one, and that it is, as such, sober. Of course, one could associate it with an understanding of distributive statements that depends on some strong ontological or epistemological condition and thus transform it into a non sober conception. Nevertheless, this is not necessary. In order to refer to such a conception saying that a certain mathematical statement $x$ is true and distinguishing between its truth and its proof or provability, it is sufficient to have at one's disposal a procedure able to decide, in any specific case covered by this statement, whether the things are or are not as claimed by the statement of $L$ equivalent to $x$ according to $R^{\prime}$ and belonging to the class $X_{t}$. In the case of the last theorem of Fermat, it is, for example, sufficient to have at one's disposal a procedure able to decide, for every set of four natural numbers $<x, y, z, n>$ satisfying the given conditions, whether $x^{n}+y^{n}=z^{n}$ or not. And there is no doubt that all who are acquainted with elementary arithmetic and have a sufficient computational capacity have such a procedure at their disposal. Of course, if the specific cases one should consider in order to exhaust the domain covered by the statement under examination are infinite in number, one would never know in this way whether this statement is true or not. But, far from being an unsatisfactory consequence of the present conception of truth, such a circumstance shows how close this conception is to any acceptable conception of truth for empirical universal statements.

\section{3}

The last conception of truth I consider here is a very classic and traditional one. I shall limit myself to observe that, under suitable conditions, this conception can be understood as being sober, and to present it in an uncustomary frame.

Let us take the example of the Bolzano-Waierstrass theorem: If $f(x)$ is a continuous function from $\boldsymbol{R}$ to $\boldsymbol{R}$ defined both in $a$ and $b, K$ is a real value, and $f(a)<K<f(b)$, then there is a real value $c$ such that $f(c)=K$.

In classical analysis, this theorem is proved by reduction to absurd, by showing that its negation contradicts the axiom of the superior upper bound. This is a non- 
constructive proof that (from a classical point of view) only warrants that $c$ exists without exhibiting it. Its legitimacy has been thus the object of several discussions. I do not want to return to such an issue here. I simply observe that the principal reason for a mathematician to be not disposed to renounce to this theorem is not a logical one, being rather concerned with the expressive power of mathematics.

A theory of real continuous function in which it is not possible to prove the Bolzano-Weierstrass theorem could hardly pretend to be useful for explaining a large class of real phenomena. Suppose that John and Mary are in Paris and that John walks from the Arc de Triomphe to the Place de la Concorde along the left side of the Champs Élysées, while Mary is somewhere on this same side of the Champs Élysées, drinking a glass of champagne. They will probably meet there. A theory or real continuous function in which it is not possible to prove the Bolzano-Waierstrass theorem cannot be used to explain this trivial phenomenon. To conclude that such a theory would thus be unsatisfactory is the same as admitting that a mathematical theory should have an expressive power and that this power is part of its mathematical legitimacy.

Once this has been admitted, it is very natural to speak of the truth of certain mathematical statements by referring to their expressive power rather than to their intra-theoric content. Let us suppose that $X$ is a class of statements of this sort belonging to a certain theory $T$ and a certain language $L$. For advancing a conception of truth which justifies thus way of speaking, it is sufficient to define a function $\varphi$ that associates any statement $x$ of $X$ with another statement $\varphi(x)$ referring to objects that are not part of the domain of $T$ and require that the function $\tau$ defined over $X$ associates any statement $x$ of $X$ to a statement of a suitable meta-language $L^{\prime}$ telling us the same as the statement $\varphi(x)$.

This is what one does when defining the truth of a mathematical statement with respect to a certain model of the theory this statement belongs to. A classic example is the definition of the truth for the statements of Peano's arithmetic with respect to one of its set-theoretical models. Notice, however, that the possibility to define the truth of a mathematical statement in this way does not depend on the possibility to define a model for the whole theory this statements belongs to according to the constraints of the logical theory of models. The statement expressing the BolzanoWeierstrass theorem could, for example, be associated by $\varphi$ to a statement concerning suitable curves traced on a Cartesian plane, without need for these curves to belong to a model (in the sense of the logical theory of models) for the whole theory of real functions.

It seems to me that in order to understand this conception of truth for a certain class $X$ of mathematical statements as being sober, it is not necessary to suppose that the statements associated to the statements of $X$ by the function $\varphi$ are not mathematical in turn, or do not refer to objects that are involved as such, or could be involved with a controversial ontology. What it is needed is simply that these latter statements or any one of their particular instances (if they are universal statements of a distributive form) are somehow decidable, that is, that there is an effective procedure to decide whether these statements or any one of their particular instances can be asserted or not. 
This last remark, together with the example given by the conceptions of truth presented above, should make clearer what I mean by "sober". Taking the Tarski's condition for granted, I maintain that a conception of truth for a class $X$ of mathematical statements of a language $L$ is sober if and only if there is no need to appeal to a controversial ontology in order to describe the conditions under which the statement $\tau(x)$ of $L^{\prime}$ is assertible.

As there is neither a generally accepted criterion to decide what is a controversial ontology nor a general accepted definition of assertibility, and my formulation of Tarski's condition does not involve any strict constraint on the nature of the statement $\tau(x)$, it would be easy to object that my characterization of the general notion of a sober conception of truth for a class of mathematical statements is so large that one could arbitrarily suggest many other sober conceptions of truth. I accept the point, but I do not think that this is an argument against my views.

I have two reasons for maintaining this.

First, it seems to me that however large it could be, my characterisation of the general notion of sober conception of truth for a class of mathematical statements sets up a general form that a sober conception of truth should satisfy. I claim this very useful because I think that the only proper way to speak of truth in general is to fix a form that a certain predicate should satisfy in order to be taken in certain contexts as the predicate "to be true." This is the same as arguing that the term "true" should be taken in general-that is, independently of any specific and contextual definitional clause-as referring to an equivalence-class of predicates rather than to a single and well-defined predicate.

My second reason is also a justification of this attitude: I argue that as far as philosophy may lead us, it cannot do more than provide us with some general categories to be used to study real phenomena. Philosophy of mathematics should provide us with some general categories that can be used to study mathematics as a given reality. Though philosophy certainly has a history, a method, and a disciplinary content to which it refers, these categories should not be shaped abstractly. What ultimately decides whether they are the good ones or not is neither philosophy nor logic. It is rather the reality to which they should be applied. 